\title{
ALTERAÇÕES MORFOMÉTRICAS DA MUSCULATURA DOS MÚSCULOS LONGITUDINAL E CIRCULAR DE RATOS SUBMETIDOS À CRIAÇÃO DE PILOROS NO INTESTINO DELGADO
}

\author{
MORFOMETRIC ALTERATIONS OF THE LONGITUDINAL AND CIRCULAR MUSCLE \\ LAYERS IN RATS SUBMITTED TO PYLORUS CONSTRUCTION IN THE SMALL \\ BOWEL
}

\author{
Cícero de Lima Rena, TCBC-MG ${ }^{\text {; Alcino Lázaro da Silva, TCBC-MG }}$; Ângela Aparecida Barra ,TCBC- MG ; \\ Maria Cristina Vasconcellos Furtado ${ }^{4}$; Rachel Lopes Rena ${ }^{5}$; Rafael Lopes Rena ${ }^{6}$
}

\begin{abstract}
RESUMO: Objetivo: Realizar um estudo morfométrico comparativo da musculatura longitudinal e circular do intestino delgado de ratos submetidos à construção cirúrgica de um e de dois piloros no intestino delgado utilizando-se da técnica de criação de piloros proposta por Rena et al. Método: Foram utilizados 52 ratos Wistar machos divididos em três grupos. O Grupo A, destacado como controle, composto de 10 animas, cada um forneceu $20 \mathrm{~mm}$ de segmento de intestino para o estudo. Os animais do Grupo B, composto de 32 animais, foram submetidos à construção de dois piloros, um a $100 \mathrm{~mm}$ e outro a $150 \mathrm{~mm}$ da válvula ileocecal e os animais do grupo $\mathrm{C}$, composto de 10 animais, foram submetidos à construção de um piloro a $100 \mathrm{~mm}$ da mesma. A eutanásia ocorreu no décimo dia. A morfometria das camadas musculares longitudinal e circular acima e abaixo dos piloros foi estudada com utilização do microscópico modelo "Axiostar plus" conectado à câmera "Axioncam Version 5.05.10" com objetiva X5 / 0,12 no o programa "AxioVision 3.1.2.1". Resultados: As alças apresentaram aumento da espessura da camada muscular acima e abaixo do ponto da operação. O estudo morfométrico comparativo das camadas musculares longitudinal e circular mostrou aumento significativo em comparação ao controle, não demonstrando significância entre os grupos B e C. Conclusão: Os resultados deste estudo revelaram importante aumento de espessura das camadas musculares, porém, menos acentuados que aqueles descritos na literatura quando foram realizados em animais submetidos à estenose fixa. Esses dados indicam que a construção operatória de piloros pela técnica utilizada promove alterações musculares de menor monta, possivelmente, pela função valvular do piloro (Rev. Col. Bras. Cir. 2007; 34(1): 41-47).
\end{abstract}

Descritores: Intestino delgado; Músculo liso; Hipertrofia; Hiperplasia; Síndrome do intestino curto.

\section{INTRODUÇÃO}

No homem e em animais de experimentos, a ressecção extensa do intestino delgado pode acarretar graves distúrbios de absorção. Há diminuição da área de absorção e/ou aumento da velocidade do trânsito levando à diarréia, desequilíbrio hidroeletrolítico e metabólico, desnutrição proteico-calórica e perda acentuada de peso caracterizando a síndrome do intestino curto (SIC).

Quando a superfície de absorção é suficiente, mas a velocidade do trânsito é aumentada, os procedimentos cirúrgicos sobre a musculatura intestinal ganharam destaque sobre outras técnicas que têm por finalidade o retardo da velocidade. A primeira proposta cirúrgica para tratamento da SIC foi de Glasmam, em 1942, quando realizou seromiectomia circunferencial em cães ${ }^{1}$. Shiller et al. realizaram, em 1967, seromiectomia da camada longitudinal ${ }^{2}$. Em 1974, Lázaro da Silva realizou seromiectomia longitudinal e circular ${ }^{3}$, circunferenciamente, para tratar pacientes portadores de SIC e dumping. Em 1982, Stacchini et al. realizaram seromiectomia da camada longitudinal, circunferencialmente, após ressecção intestinal extensa em cães ${ }^{4}$. Em 1986, Stacchini et al. utilizando-se da seromiectomia da camada longitudinal puderam comprovar a diminuição da velocidade do trânsito intestinal por meio radiológico 5 . Em 1996, Rena et al. propuseram, para tratar pacientes portadores de SIC, a criação cirúrgica de um piloro pelo sepultamento de um anel seromuscular de $2 \mathrm{~cm}$ de extensão entre duas seromiotomias ${ }^{6}$. Esse procedimento demonstrou bons resultados e revelou retardo do trânsito intestinal ao estudo radiológico.

O objetivo do experimento foi analisar, quantitativamente, a morfometria dos músculos longitudinal

1. Professor Adjunto IV de Cirurgia da Faculdade de Medicina da Universidade Federal de Juiz de Fora; Mestre em Técnica Cirúrgica e Cirurgia Experimental; Doutorando em Cirurgia na UFMG.

2. Professor Titular de Cirurgia da Faculdade de Medicina da Universidade Federal de Minas Gerais.

3. Cirurgiã Geral da Santa Casa de Misericórdia de Juiz de Fora; Mestranda em Cirurgia na UFMG.

4. Professora de Anatomia da Universidade Federal de Juiz de Fora; Cirurgiã Geral da Santa Casa de Misericórdia da Santa Casa de Misericórdia de Juiz de Fora; Doutoranda em Cirurgia na UFMG.

5. Cirurgiã e Coloproctologista da Santa Casa de Misericórdia de Juiz de Fora.

6. Estagiário de Medicina da Faculdade de Medicina de Vassouras R J.

Recebido em 02/08/06

Aceito para publicação em 01/09/06

Conflito de interesses: nenhum

Fonte de financiamento: nenhuma

Trabalho realizado no Instituto de Biologia da Reprodução da Universidade Federal de Juiz de Fora e Pós-Graduação de Cirurgia da Universidade Federal de Minas Gerais. 
e circular pré e pós-pilóricos no intestino delgado de ratos submetidos à criação cirúrgica de piloros intestinais pela técnica de Rena et al ${ }^{6}$. Os trabalhos experimentais publicados referem-se a estudos morfométricos da musculatura intestinal quando se utiliza de estenose cirúrgica fixa e não com sistema valvular como proposto pelos autores.

\section{MÉTODO}

Foram utilizados 52 ratos (Rattus norvegicus, Berkenhout,1769), originalmente Wistar, com cinco meses de idade, machos,pesando entre $220 \mathrm{~g}$ e $354,29 \mathrm{~g}$ criados pelo Biotério do Centro de Biologia de Reprodução da Universidade Federal de Juiz de Fora. Não houve óbitos.

Os animais foram distribuidos em três grupos. O Grupo A composto de 10 animais, correspondendo ao grupo controle, o Grupo B formado por 10 animais nos quais foram construídos, em cada animal, dois piloros e um grupo C constituído por 32 animais nos quais, em cada animal, foi construído um piloro.

Os animais foram submetidos a jejum de $12 \mathrm{~h}$ para o procedimento cirúrgico. Antes da operação era feita a pesagem, a seguir a tricotomia na região ventral, com utilização de aparelho elétrico específico para este fim. A área a ser operada era limpa e desinfetada com álcool a 70\%. Os animais recebiam a combinação de $10 \mathrm{mg} / \mathrm{kg}$ de xilazina $+90 \mathrm{mg} /$ $\mathrm{kg}$ de ketamina intraperitoneal. Após a constatação do estado de anestesia cirúrgica, os animais eram submetidos ao procedimento.

Uma incisão abdominal mediana, de aproximadamente $20 \mathrm{~mm}$, com lâmina de bisturi número 15 , iniciava-se na linha mediana, terço distal da parede, abrangendo pele e tela subcutânea. Utilizando-se uma tesoura, os músculos eram separados na linha mediana e a cavidade peritoneal era aberta. Para facilitar a visibilização, uma lupa de aumento de quatro vezes foi empregada.

Nos animais do grupo A, a partir de $100 \mathrm{~mm}$ da junção ileocecocólica, foi retirado de cada animal, um segmento do intestino de $20 \mathrm{~mm}$ de comprimento para estudo controle.

Nos animais do Grupo B, a $100 \mathrm{~mm}$ da junção ileocecocólica, foram realizadas duas seromiotomias circunferenciais, abrangendo as camadas serosas e musculares, separadas por $2 \mathrm{~mm}$ de parede seromuscular intestinal íntegra. A borda cruenta proximal da seromiotomia cranial foi suturada à borda cruenta distal da seromiotomia caudal com pontos separados de ácido poliglicólico 5.0. Assim, construíase um primeiro piloro a 100mm da junção (Figura1 - pág. 43). A $50 \mathrm{~mm}$ deste, um novo piloro foi construído. A este procedimento denominou-se seromiotomia dupla com criação de um piloro em dois locais no intestino delgado.

Nos animais do Grupo C, o mesmo procedimento foi repetido a $100 \mathrm{~mm}$ da junção íleocecocólica. A este procedimento, deu-se o nome de seromiotomia dupla com criação de um piloro. Em todos os animais a parede abdominal foi fechada em dois planos, por suturas contínuas, com fio de seda 3.0.

Na primeira noite pós-operatória, os animais recebiam ração umedecida em água. Com a melhora da aceitação da dieta, esta era substituída por ração seca.
Após as operações, os animais foram mantidos em gaiola e ambiente apropriados.

No décimo dia pós-operatório, os animais foram submetidos à eutanásia para retirada do material para estudo. Após a tricotomia, quando necessária, os animais foram anestesiados da mesma forma que para a cirurgia. Em seguida eram submetidos à laparotomia para coleta das peças para os estudos. Após a retirada do material, a anestesia era aprofundada até causar a morte do animal ${ }^{7}$.

Foi realizada a exerese dos piloros e segmentos intestinais de, aproximadamente, $10 \mathrm{~mm}$ cranial e distalmente aos mesmos. Os segmentos intestinais eram abertos na face antimesentérica (Figura 2 - pág. 43) e fixados com grampo a um suporte de isopor e acondicionados em recipientes com formol a $10 \%$.

A obtenção do material para estudo à microscopia óptica de luz seguiu as etapas da técnica histológica convencional.

Para visibilização dos componentes teciduais, realizou-se coloração pela hematoxilina-eosina e tricrômico Gomori. A montagem foi feita em lâminas em bálsamo do Canadá.

Para o estudo morfométrico, foi utilizado microscópico modelo "Axiostar plus" conectado à câmera "Axioncam Version 5.05.10" com objetiva X5 / 0,12 no o programa "AxioVision 3.1.2.1" nos laboratórios do Centro de Biologia da Reprodução da Universidade Federal de Juiz de Fora.

Para análise estatística foram utilizados os testes de Pearson, Shapiro wilk, Levene, Teste t, Anova e post-hoc LSD) e Tukey.

Este trabalho está de acordo com os Princípios Éticos na Experimentação Animal adotado pelo Colégio Brasileiro de Experimentação Animal (COBEA) e foi aprovado pela Comissão de Ética na Experimentação Animal (CEEA) do Centro de Biologia da Reprodução da UFJF, em reunião realizada em 07 de fevereiro de 2002.

\section{RESULTADOS}

Interessou ao estudo a medida da espessura da camada da musculatura longitudinal e circular do segmento envolvido no experimento.

\section{GRUPOA}

Identificado como grupo controle, não foi submetido ao procedimento cirúrgico do experimento. O estudo das medidas da espessura da camada muscular longitudinal e circular no segmento estudado prestou-se como padrão de controle.

O músculo longitudinal $(\mathrm{ml})$ teve como medida de menor valor $12,47 \mu \mathrm{m}$ e de maior valor $56,89 \mu \mathrm{m}$ com média de $32,96 \mu \mathrm{m}$ e mediana de $35,59 \mu \mathrm{m}$.

O músculo circular $(\mathrm{mc})$ apresentou medida que variou de $45,27 \mu \mathrm{m}$ a $118,01 \mu \mathrm{m}$, tendo como média $63,57 \mu \mathrm{m}$ e como

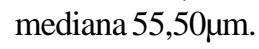

\section{GRUPOB}

Submetido à construção de dois piloros.

Foram medidas as espessuras dos músculos longitudinais e circulares pré-pilóricos proximais e distais e póspilóricos proximais e distais. 


\section{Piloro proximal}

As medidas de espessura do músculo longitudinal pré-pilórico proximal variaram de $42,07 \mu \mathrm{m}$ a $105,43 \mu \mathrm{m}$, com

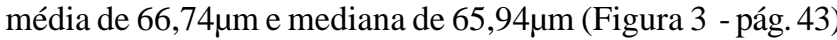
As medidas do músculo pós-pilórico proximal foram de $37,65 \mu \mathrm{m}$ a $119,59 \mu \mathrm{m}$ com média de $77,32 \mu \mathrm{m}$ e mediana de

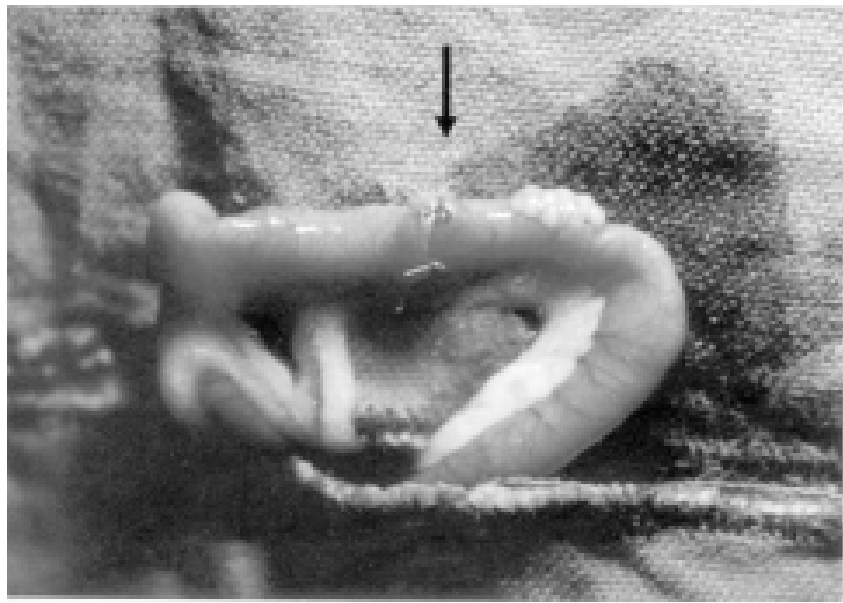

Figura 1 - Piloro construído a 100 mm da válvula ileocecal.



Figura 2 - Abertura da alça no sentido longitudinal mostrando o piloro (seta).

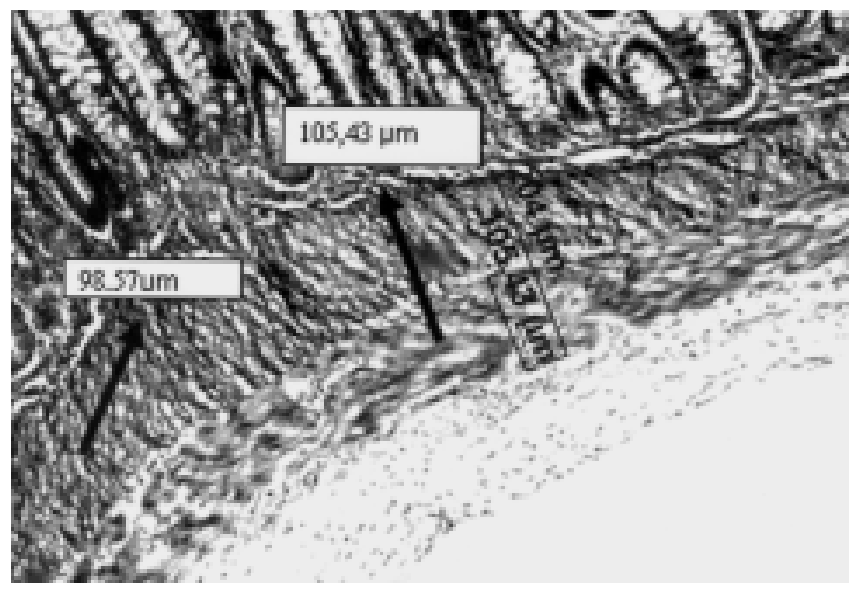

Figura 3 - Morfometria do músculo longitudinal pré-pilórico do piloro proximal (mlprépp) 105,43 $\mu \mathrm{m}$ e morfometria do músculo circular pré-pilórico do piloro proximal (mcprépp) 98,57 $\mu \mathrm{m}$ do animal de número 6 (grupo 2) Triocrômio Gomori X.
$75,29 \mu \mathrm{m}$, A espessura do músculo circular pré-pilórico foi de $71,13 \mu \mathrm{m}$, na menor medida e de $159,49 \mu \mathrm{m}$ na maior tendo como média $122,68 \mu \mathrm{m}$ e como mediana $123,88 \mu \mathrm{m}$. O músculo circular pós-pilórico mediu 38,12 $\mu \mathrm{m}$ na menor espessura e $222,28 \mu \mathrm{m}$ na maior, tendo como média $122,62 \mu \mathrm{m}$ e como mediana $120,46 \mu \mathrm{m}$.



Figura 4 - Morfometria do músculo longitudinal pós-pilórico do piloro distal (mlppd) 79,12 4 m e morfometria do músculo circular pós-pilórico do piloro distal (mcppd) 157,53 $\mu \mathrm{m}$ do animal de número $6 \mathrm{HE} X$.

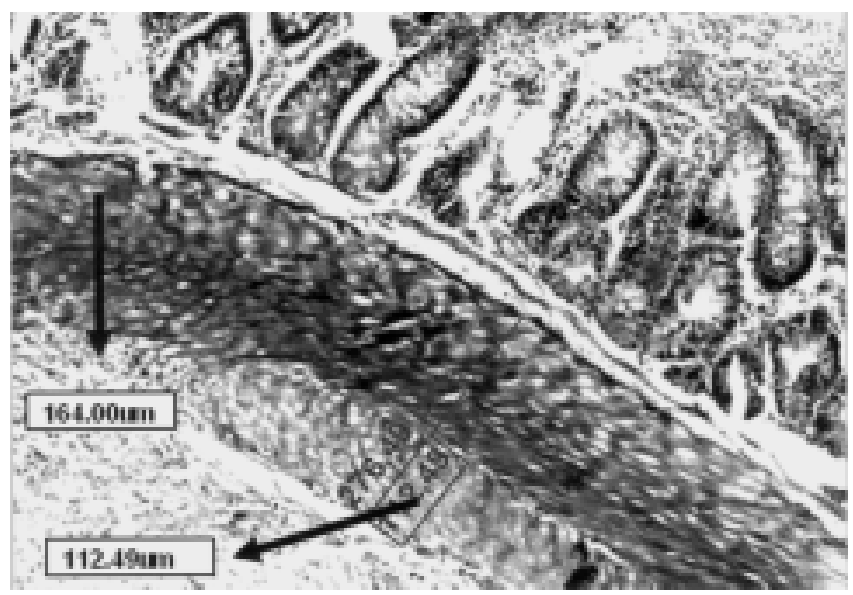

Figura 5 - Morfometria do músculo longitudinal pré-pilórico (mlprép) 112,49 нm e morfometria do músculo circular pré-pilórico (mcppd) 164,00 $\mu m$ do animal de número 1.

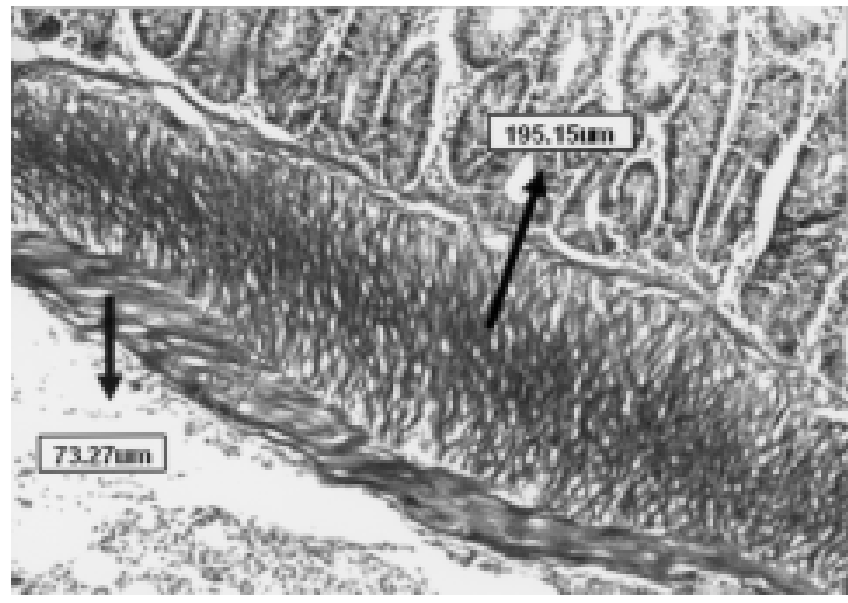

Figura 6 - Morfometria do músculo longitudinal pós-pilórico (mlpósp) 73,27 нm e morfometria do músculo circular pós-pilórico (mcpósp) 195,15 um do animal de número 1 do grupo $C$. 
Tabela 1 - Comparações de medidas do grupo controle com os grupos B e $C$.

\begin{tabular}{|c|c|c|c|c|}
\hline Comparaçãodemedidas & Pressupostos & & & $\begin{array}{l}\text { Comparação de } \\
\text { duas médias }\end{array}$ \\
\hline & Correlação & Normalidade & Igualdade de variâncias & Nãopareado \\
\hline \multicolumn{5}{|l|}{ MORFOMÉTRICAS } \\
\hline A X B eC & $p$ & $p$ & $p$ & $p$ \\
\hline \multicolumn{5}{|l|}{ longitudinal } \\
\hline mlAx mlpC & 0,672 & $\begin{array}{l}0,360 \\
0,920\end{array}$ & 0,370 & 0,001 \\
\hline mlAx mlpoC & 0,454 & 0,293 & 0,625 & 0,155 \\
\hline mlA x mlpppB & 0,545 & 0,606 & 0,937 & 0,0001 \\
\hline mlA x mlpoppB & 0,801 & 0,815 & 0,623 & 0,004 \\
\hline mlA x mlppdB & 0,473 & 0,849 & 0,250 & 0,0001 \\
\hline mlA x mlpopdB & 0,020 & 0,090 & 0,110 & 0,0001 \\
\hline \multicolumn{5}{|l|}{ circular } \\
\hline mcA x mcppC & 0,691 & $\begin{array}{l}0,110 \\
0,285\end{array}$ & 0,120 & 0,0001 \\
\hline mcA x mcpopC & 0,300 & 0,511 & 0,173 & 0,0001 \\
\hline mcA x mcpppB & 0,558 & 0,068 & 0,257 & 0,004 \\
\hline mcA x mсрорpB & 0,097 & 0,534 & 0,127 & 0,003 \\
\hline mcA x mcppdB & 0,145 & 0,735 & 0,965 & 0,0001 \\
\hline mcA x mcpopdB & 0,076 & 0,567 & 0,153 & 0,0001 \\
\hline
\end{tabular}

mlA $x$ mlpC-musculatura longitudinal pré-pilórico do grupo $C$

mlAx mlpoC-musculatura longitudinal pós-pilórico do grupo $C$

mlAx mlpppB - musculatura longitudinal pré-pilórico proximal do grupo $B$

mlAx mlpopp $B$ - musculatura longitudinal pós-pilórico proximal do grupo $B$

mlAx mlppdB - musculatura longitudinal pré-pilórico distal do grupo $B$

mlAx mlpopdB - musculatura longitudinal pós-pilórico distal do grupo $B$

mсA х тсррC- musculatura circular pré-pilórico do grupo $C$

mcA х тсрорC - musculatura circular pós-pilórico do grupo $C$

mсA х тсррpB - musculatura circular pré-pilórico proximal do grupo $B$

тсA $х$ тсроррB - musculatura circular pós-pilórico proximal do grupo $B$

$m c A x$ mсррdB - musculatura circular pré-pilórico distal do grupo $B$

mcA х mсрорdB - musculatura circular pós-pilórico distal do grupo $B$

\section{Piloro distal}

A medida da espessura do músculo longitudinal prépilórico teve por menor medida $25,28 \mu \mathrm{m}$ e por maior medida $122,29 \mu \mathrm{m}$, apresentando como média $66,88 \mu \mathrm{m}$ e como mediana $63,12 \mu \mathrm{m}$. A espessura do músculo pós-pilórico variou de $45,77 \mu \mathrm{m}$ a $82,76 \mu \mathrm{m}$ apresentando uma média de $71,17 \mu \mathrm{m} \mathrm{e}$

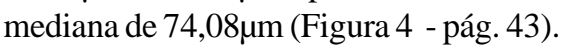

O músculo circular pré-pilórico teve medidas variando de $74,16 \mu \mathrm{m}$ a $128,01 \mu \mathrm{m}$, apresentando média de $111,72 \mu \mathrm{m}$ e mediana de $112,26 \mu \mathrm{m}$. As medidas do músculo no segmento pós-pilórico foram de $87,39 \mu \mathrm{m}$ na menor e de $202,41 \mu \mathrm{m}$ na

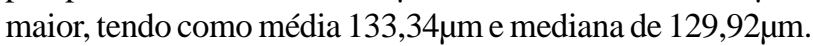

\section{GRUPOC}

Animais submetidos à construção de um piloro.

O músculo longitudinal pré-pilórico teve medida que variou de $23,69 \mu \mathrm{m}$ a $112,49 \mu \mathrm{m}$ com média de $67,79 \mu \mathrm{m}$ e media-

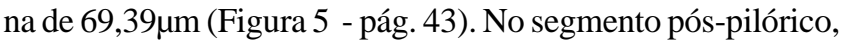
as medidas foram de $23,69 \mu \mathrm{m}$ a $125,08 \mu \mathrm{m}$, tendo como média

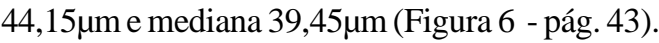

O músculo circular pré-pilórico apresentou medidas que variaram de $57,67 \mu \mathrm{m}$ a $262,97 \mu \mathrm{m}$, com média de $130,51 \mu \mathrm{m}$ e mediana de $132,04 \mu \mathrm{m}$. No segmento pós-pilórico, as medidas variaram de $44,61 \mu \mathrm{m}$ a $282,18 \mu \mathrm{m}$, tendo por média $129,24 \mu \mathrm{m}$ e mediana de $119,14 \mu \mathrm{m}$.

\section{DISCUSSÃO}

A hipertrofia da musculatura lisa intestinal foi descrita por Herezel em 1886 como sendo conseqüente à dificuldade de trânsito intestinal em um caso de obstrução do intestino delgado ${ }^{8}$. Observou-se que este aumento de espessura da camada muscular ocorria acima do processo obstrutivo e este fenômeno foi denominado de hipertrofia compensatória. Foi o estímulo ao estudo dos músculos liso longitudinal e circular do intestino delgado e músculos de outras vísceras ocas. Esses estudos abrangeram diversos órgãos e situações variadas. Os músculos ureterais, vesicais, uterinos e em vários níveis do sistema digestivo foram estudados por vários pesquisadores ${ }^{9-12}$. $\mathrm{O}$ interesse 
Tabela 2 - Comparação de medidas morfométricas entre os grupos B e C.

\begin{tabular}{llll}
\hline Comparações de Medidas & Pressupostos & & Comparação de 2 médias \\
\hline 1.MORFOMÉTRICAS & Correlação & Igualdade de variânçia & Pareado \\
(B X C) & $\boldsymbol{p}$ & $\boldsymbol{p}$ & $\boldsymbol{p}$ \\
\hline Longitudinal & & & \\
mlpC x mlppB & 0,368 & 0,468 & 0,916 \\
mlpC x mlppdB & 0,016 & 0,727 & 0,937 \\
mlpC x mlpoppB & 0,790 & 0,775 & 0,380 \\
mlpC x mlpopdB & 0,925 & 0,057 & 0,691 \\
mlpoC x mlpppB & 0,807 & 0,776 & $0,013(* *)$ \\
mlpoC x mlppdB & 0,044 & 0,895 & 0,002 \\
mlpoC x mlpoppB & 0,895 & 0,360 & $0,044(* *)$ \\
mlpoC x mlpopdB & 0,720 & 0,035 & 0,001 \\
Circular & & & \\
mcpC x mcppB & 0,624 & 0,633 & 0,589 \\
mcpC x mcppdB & 0,436 & 0,100 & 0,150 \\
mcpC x mcpoppB & 0,491 & 0,591 & 0,682 \\
mcpC x mcpopdB & 0,310 & 0,957 & 0,859 \\
mcpoC x mcpppB & 0,385 & 0,710 & 0,656 \\
mcpoC x mcppdB & 0,116 & 0,572 & 0,733 \\
mcpoC x mcpoppB & 0,092 & 0,151 & 0,187 \\
mcpoC x mcpopdB & 0,577 & 0,911 & 0,811 \\
\hline
\end{tabular}

mlpC-musculatura longitudinal pré-pilórica do grupo $C$

mlpoC - musculatura longitudinal pós-pilórica do grupo $C$

mlppB- musculatura longitudinal pré-pilórica do piloro proximal do grupo $B$

mlppdB - musculatura longitudinal pré-pilórica do piloro distal do grupo $B$

mlpopp $B$ - musculatura longitudinal pós-pilórica do piloro proximal do grupo $B$

mlpopdB-musculatura longitudinal pós-pilórica do piloro distal do grupo $B$

mсрC - musculatura circular pré-pilórica do grupo $C$

mсроC - musculatura circular pós-pilórica do grupo $C$

mсррB - musculatura circular pré-pilórica do piloro proximal do grupo $B$

mсррdB - musculatura circular pré-pilórica do piloro distal do grupo $B$

тсрорр $B$ - musculatura circular pós-pilórica do piloro proximal do grupo $B$

mсрорdB - musculatura circular pós-pilórica do piloro distal do grupo $B$

maior foi despertado pelo intestino delgado em virtude do seu envolvimento, principalmente em enfermidades obstrutivas ou isquêmicas que podem trazer, como conseqüência, perdas importantes de áreas de absorção causadas pelas ressecções extensas. Os enfermos, em número expressivo, necessitam de um período de adaptação do intestino remanescente para que a absorção de nutrimentos sustente a vida. Alguns pacientes, todavia, desenvolvem a síndrome do intestino curto. $\mathrm{O}$ advento da nutrição parenteral total (NPT), inclusive domiciliar, trouxe uma grande ajuda aos necessitados embora não seja isenta de complicações. Quando, com todos os recursos disponíveis, não é possível a recuperação da ingestão pela via oral, os cirurgiões são chamados a colaborar nesse desafio. Os procedimentos cirúrgicos propostos têm por finalidade aumentar a área de absorção ou diminuir a velocidade do trânsito intestinal.

No presente experimento, o objetivo foi estudar a morfometria da musculatura lisa longitudinal e circular após o procedimento de Rena e cols., acima e abaixo dos piloros ${ }^{6}$.
É sabido que o processo obstrutivo leva à estase do conteúdo da alça a montante. A musculatura do intestino responde com aumento de volume da célula muscular, causando a hipertrofia, e, responde também, com aumento do número de células musculares tendo, como conseqüência levando a hiperplasia ${ }^{12}$.O estudo, no $10^{\circ}$ dia pós-operatório justificouse por ser o momento em que o aumento da espessura da camada muscular atinge o seu volume máximo graças à atividade de neo-síntese de DNA ${ }^{13}$.

Os testes estatísticos foram realizados usando-se as médias dos resultados. Foram analisados os três grupos tendo, como controle, o grupo A que apresentou a camada muscular longitudinal com uma média de 32,96um e a circular com $63,57 \mu \mathrm{m}$. Os Grupos B e C foram comparados ao controle e entre si (Tabela 1, Tabela 2, Tabela 3).

É aceito pela comunidade científica que o aumento da espessura da parede da alça intestinal ocorre graças à hipertrofia celular e à hiperplasia da célula muscular ${ }^{13,14}$. A possibilidade da célula muscular sofrer duplicação foi uma conquista recente da pesquisa ${ }^{13}$. A célula muscular vai à mitose 
Tabela 3 - Comparações morfométricas entre os grupos AX B X C.

\begin{tabular}{|c|c|c|c|c|c|}
\hline \multirow[t]{2}{*}{ Comparação de medidas } & \multicolumn{2}{|c|}{ Comparação de medidas } & \multicolumn{3}{|c|}{ Testes paramétricos para comparação de médias } \\
\hline & \multicolumn{2}{|c|}{ Igualdade de variança } & \multicolumn{3}{|c|}{ Comparação de 3 médias } \\
\hline 2.MORFOMÉTRICAS & & Anova & Testes & & \\
\hline$A \times B \times C$ & & & $\mathbf{A} \times \mathbf{B}$ & $\mathrm{AxC}$ & $\mathbf{B} \times \mathbf{C}$ \\
\hline & $\mathbf{p}$ & $\mathbf{p}$ & $\mathbf{p}$ & $\mathbf{p}$ & $\mathbf{p}$ \\
\hline \multicolumn{6}{|l|}{$\overline{\text { Longitudinal }}$} \\
\hline mlA x mlpC x mlpppB & 0,605 & 0,001 & 0,001 & 0,001 & 0,907 \\
\hline mlA x mlpCx mlppdB & 0,502 & 0,003 & 0,003 & 0,003 & 0,931 \\
\hline mlA xmlpCx mlpoppB & 0,624 & 0,001 & 0,001 & 0,001 & 0,997 \\
\hline mlA x mlpC x mlpopdB & 0,098 & 0,0001 & 0,0001 & 0,0001 & 0,677 \\
\hline mlA x mlpoC x mlpppB & 0,917 & 0,001 & 0,001 & 0,166 & $\begin{array}{l}0,008 \\
\text { (LSD) } \\
0,021 \\
\text { (Tukey) }\end{array}$ \\
\hline mlA x mlpoC x mlppdB & 0,398 & 0,005 & 0,005 & 0,251 & $\begin{array}{l}0,025 \\
\text { (LSD) } \\
0,062 \\
\text { (Tukey) }\end{array}$ \\
\hline mlAx mlpoC x mlpoppB & 0,575 & 0,002 & 0,002 & 0,211 & 0,012 \\
\hline mlAx mlpoC x mlpopdB & 0,084 & 0,0001 & 0,0001 & 0,111 & 0,0001 \\
\hline $\begin{array}{l}\text { Circular } \\
\text { mcA x mcpC x mcppB }\end{array}$ & 0,269 & 0,0001 & 0,0001 & 0,0001 & 0,549 \\
\hline mcA x mcpC x mcppdB & 0,147 & 0,0001 & 0,0001 & 0,0001 & 0,117 \\
\hline mcA x mcpC x mсpoppB & 0,222 & 0,001 & 0,001 & 0,0001 & 0,635 \\
\hline mcA x mcpC x mcpopdB & 0,250 & 0,0001 & 0,0001 & 0,0001 & 0,841 \\
\hline mcA x mcpoC x mcpppB & 0,340 & 0,0001 & 0,0001 & 0,0001 & 0,620 \\
\hline mcA x mcpoC x mcppdB & 0,216 & 0,0001 & 0,0001 & 0,0001 & 0,150 \\
\hline mcA xmcpoC x mсроppB & 0,245 & 0,001 & 0,001 & 0,0001 & 0,693 \\
\hline mc1x mcpoC x mcpopdB & 0,298 & 0,0001 & 0,0001 & 0,0001 & 0,775 \\
\hline
\end{tabular}

mlA - musculatura longitudinal do grupo controle

mcA - musculatura circular do grupo controle

simples ou, através da diferenciação dos fibroblastos situados na linha de transição entre a submucosa e a camada muscular, em células musculares levando ao aumento do número delas, fato que é auxiliado pelo estímulo do hormônio de crescimento ${ }^{15}$.

No presente experimento, em que o procedimento proposto foi a criação de um piloro semelhante ao descrito por Rena et al. ${ }^{6}$ não houve o propósito de causar suboclusão intestinal, o que foi verificado pela inexistência de óbitos e, macroscopicamente, durante a retirada das peças, mas de criar um sistema valvular com mecanismo de abertura e fechamento. Foi verificado que a espessura da parede muscular longitudinal e circular ultrapassou de duas vezes a mesma variável do grupo controle. Em trabalhos experimentais com estenose cirúrgica esse aumento na espessura da camada muscular foi superior a 10 vezes ${ }^{12,16,17}$. Pode-se afirmar que a construção do procedimento proposto por Rena et $a l^{6}{ }^{6}$ não apresentou diferença significante quanto ao aumento da espessura da musculatura longitudinal e circular quando estão presentes um piloro ou dois piloros.

- O estudo demonstrou que a espessura das camadas musculares longitudinal e circular apresentou aumento significante. O aumento verificado não ultrapassou de 2,09 vezes o valor do controle para o músculo circular e de 2,34 vezes para o longitudinal contra um aumento descrito na literatura de mais de 10 vezes quando se usa o modelo com extenose fixa. Esses dados indicam que a construção cirúrgica de piloros pela técnica estudada promove alterações musculares de menor monta, ocasionados possivelmente, pela função valvular do piloro.

- Novos estudos, serão necessários para melhor avaliação do procedimento. 


\begin{abstract}
Background and Purpose: To undertake a morphometric comparative study of the longitudinal and circular layers of the small bowel muscularis propria in rats submitted to surgical constructions of one and two pylorus in the small bowel, utilizing the two pylorus technique described by Rena et al. Methods: 52 male Wistar rats were divided into three groups. Group A (10 animals), was defined as the control group, had $20 \mathrm{~mm}$ of the bowel segment removed for the study. Group B (10 animals) underwent construction of two pylorus, the first at $100 \mathrm{~mm}$ and the second at $150 \mathrm{~mm}$ from the ileo-cecal valve. Animals from group C(32 animals), underwent construction of one pylorus at $100 \mathrm{~mm}$ from the ileo-cecal valve. The rats were sacrificed ten days after the procedure. The morphometric analysis of the longitudinal and circular layers of the muscularis propria above and below the pylorus were studied with a microscope Axiostar Plus model connected to the Axioncam camera version 5.05.10 with 5x/0,12 objective utilizing the software Axion Vision 3.1.2.1. Results: The bowel loops demonstrated thickening of the muscular layer in segments above and below the point where the surgical procedure was performed. The morphometric comparative study of the longitudinal and circular muscular layers demonstrated significant increase when compared to the control group. There were no significant differences among groups $B$ and C. Conclusion: The results showed a significant increase in muscle layers, however, less than the increase that has been reported in the literature for animals that underwent fixed stenosis. These data indicates that surgical construction of the pylorus by the technique used by us promotes muscular changes of minor significance, possibly, by the valvular function of the pylorus.
\end{abstract}

Key words: Intestine, small; Muscle, smooth; Hypertrophy; Hyperplasia; Short bowel syndrome.

\section{REFERÊNCIAS}

1 Glassmam JA. An artificial ileocecal valve. Surg Gynecol Obstet. 1942; 74(1):92-8.

2 Schiller WR, DiDio LJ, Anderson MC. Production of artificial sphincters. Ablation of the longitudinal layer of the intestine. Arch Surg. 1967; 95(3):436-42.

3 Lázaro da Silva A. Tentativa de tratamento do "dumping" através de um "esfincter" ileal. Rev Assoc Med Minas Gerais. 1974; 25(1):32-3.

4 Stacchini A, DiDio LJ, Primo ML, Borelli V, Andretto R. Artificial sphincters as surgical treatment for experimental massive resection of small intestine. Am J Surg. 1982; 143(6):721-6.

5 Stacchini A, DiDio LJ, Christoforidis AJ, Borelli V. Intestinal transit time is delayed by artificial sphincters after massive enterectomy in dogs. Am J Surg.1986; 151(4):480-83.

6 Rena CL, Lázaro da Silva A, Barra AA, Melo GE, Paula WT. Seromiotomia dupla no intestino delgado: tentativa de criação de um piloro artificial. Rev Col Bras Cir. 1996; 23(3):143-7.

7 Herezel E. Experimentelle und histologische untersuchungen der compensatorischen muskelhypertrophie bei darmstenosen. Klin Med 1886; 11:321-54.

8 Lange KH. Uber die hipertrophie der glatten musculature. Morfol Jahrb 1940; 84:363-402.

9 Cussen LJ, Tymms A. Hyperplasia of urethral muscle in response to acute obstruction of the ureter. Invest Urol. 1972; 9(5):504-8.

10 Gee WF, Kiviat MD. Ureteral response to partial obstruction. Smooth muscle hyperplasia and connective tissue proliferation. Invest Urol. 1975; 12(4):309-16.

11 Gabella G. Hypertrophy of intestinal smooth muscle. Cell Tissue Res. 1975; 163(2):199-214.

12 Gabella G. Hypertrophic smooth muscle. I. Size and shape of cells, occurrence of mitoses. Cell Tissue Res. 1979; 201(1): 6378.
13. Gabella G. Hypertrophic smooth muscle. Size and Shape of cell, occurrences of mitoses. Cell Tiss Res 1979; 201: 63 - 78.

14. Brossa O, Geuna S, Poncino A, Seccia M, Giacobini-Robecchi MG, Gravela E. The partial intestinal stenosis: An evaluation of smooth muscle hypertrophy, hyperplasia and omithine decarboxylase (ODC) activity in the loops upstream from a surgical obstruction. Riv Ital Biol Med 1992a; 12: 14 - 17.

15. Vinter-Jensen L, Juhl CO, Dajani EZ, Niesen K, Djurhuus JC. Chronic systemic treatment with epidermal growth factor induces smooth muscle cell hyperplasia and hypertrophy in the urinary tract of mature Goettingen minipigs. Br J Uro11997; 79: 532 38.

16. Canavese M, Geuna S, Poncino A, Giacobini-Robeceti MG Iperplasia del tessuto muscolare liscio intestinale a monti di una steonsi chirurgica parziale: Uno studio autoradiografico. Boll Soc Ital Biol 1992; 68: 9 -16.

17. Geuna S, Cardillo S, Giacobini-Robecchi MG. Smooth Cell Hypertrophy and Hyperplasia in the Partially Obstructed Gut of the Rat: A Quantitative Evaluation. Acta Anat 1998; 163: 69 74.

Como citar este artigo

Rena CL, Lázaro da Silva A, Barra AA, Furtado MCV, Rena RL, Rena RL. Alterações morfométricas dos músculos longitudinal e circular em ratos submetidos à criação de piloros no intestino delgado. Rev Col Bras Cir. [periódico na Internet] 2007;34(1). Disponível em URL: http://www.scielo.br/rcbc

Endereço para correpondência:

Cícero de Lima Rena

Rua Kátia Manfrói, 205

Cidade Jardim

36026-560 - Juiz de Fora - MG

E-mail - cicero.rena@ superig.com.br 